\title{
Risk of permanent pacemaker implantation after transcatheter aortic valve replacement: How do we manage beyond risk assessment?
}

\author{
Alex Velasquez ${ }^{1}$ and Jeffrey Goldberger ${ }^{2}$ \\ ${ }^{1}$ University of Miami Miller School of Medicine \\ ${ }^{2}$ University of Miami
}

November 2, 2021

Risk of permanent pacemaker implantation after transcatheter aortic valve replacement: How do we manage beyond risk assessment?

\author{
Alex Velasquez, $\mathrm{MD}^{1}$ \\ Jeffrey J. Goldberger, MD, MBA ${ }^{1}$ \\ 1 Cardiovascular Division, Department of Medicine, University of Miami Leonard M. Miller School of \\ Medicine, Miami, FL
}

\section{Corresponding author:}

Jeffrey J. Goldberger, M.D.

University of Miami Miller School of Medicine

Cardiovascular Division

$1120 \mathrm{NW} 14^{\text {th }}$ St.

Miami, FL 33136

Telephone: (305) 243-8092

Fax: (305) 243-1731

E-mail: j-goldberger@miami.edu

Aortic stenosis (AS) is the most common valve disease worldwide, with degenerative (calcific) AS being the most common etiology in western countries. Patients experience a high morbidity and mortality without intervention to correct the mechanical obstruction to cardiac output ${ }^{1}$. Since 1960, when Dwight Harken performed the first in-situ aortic valve replacement with a ball-in-cage mechanical valve, cardiothoracic surgeons have been dramatically improving patients' lives ${ }^{2}$. The implications of how we treat this condition will become amplified by an aging US population and an increased incidence of AS with age. ${ }^{3}$ At its inception, surgical aortic valve replacement was recognized to have trade-offs including injury to the native atrioventricular(AV) conduction system ${ }^{4}$. The AV conduction system of the heart resides in such close anatomic proximity to the aortic valve that injury is often encountered. As surgical techniques improved, the rate of significant post-operative heart block that requires permanent pacemaker implantation (PPMI) decreased and now hovers between $3.3-6.9 \%$. ${ }^{5-7}$ 
In the last two decades, patients deemed poor surgical candidates were offered an opportunity of similar improvements in mortality and quality of life when trans-catheter techniques for valve replacement were developed. The low morbidity and mortality associated with these techniques have driven the medical community to consider these therapies for moderate and even low risk surgical cases. ${ }^{7}$ As the number of patients who receive this life-saving therapy increases, so too will its most common complication occur.

Between 10-25\% of patients that receive transcatheter aortic valve replacement (TAVR) undergo PPMI. Understanding the pre-operative and intra-operative case characteristics that are associated with PPMI is so popular that Wang et al. was able to pool a total of 97,294 patients in 67 retrospective and prospective studies that examine this subject. In this meta-analysis they concluded that pre-existing cardiac conduction abnormality and trans-femoral pathway selection were significantly associated with PPMI. Pre-existing left bundle branch block, patient-prosthesis mismatch, and implantation depth were not. ${ }^{8}$

While the information provided in this meta-analysis is valuable, the main question confronting the clinician managing a patient following TAVR is whether the patient needs to have a PPMI. In the patient with persistent complete or high-grade AV block, the clinical decision is easy. Similarly, in those without preexisting conduction system disease and in those with no change in their pre-existing conduction system disease post-TAVR, there is consensus that pacing is not required. The challenge and management disparity lie among those with transient severe conduction abnormalities and those with significant changes in native conduction, including significant PR interval prolongation and those with new left bundle branch block. Anatomically, there may be considerable variation in the location of the AV conduction system and left bundle in the proximity to the aortic valve ${ }^{9}$. Unfortunately, this major anatomic determinant of the risk of AV block cannot be assessed or imaged prior to the procedure. The surface ECG provides important clues. While striking changes in the QRS complex, ie the development of left bundle branch block, are routinely recognized and addressed, subtler changes in the PR interval may be overlooked. The PR interval has 3 components - intratrial conduction time from the sinus node to the AV node, AV nodal conduction time, and His-Purkinje system conduction time. A $50 \mathrm{~ms}$ increase in the PR interval from 140 to $190 \mathrm{~ms}$, for example, might still be considered normal when reviewed in isolation, but a $50 \mathrm{~ms}$ increase in His-Purkinje system conduction time could be a harbinger of risk for complete AV block. Interestingly, the meta-analysis reported an enhanced risk for PPMI in patients with first degree AV block but not those with second degree AV block. A physiologic explanation is that in patients with first degree AV block much of this delay may be in the His-Purkinje system, while in those with second degree AV block (if it is Wenckebach) this is a manifestation of AV nodal delay and not considered pathologic. It is therefore critical to evaluate serial changes in conduction post-TAVR and follow expert panel guidance on management ${ }^{10}$. Electrophysiologic testing is sometimes necessary to assess the integrity of the conduction system and to decide whether a pacemaker is required (see figure) ${ }^{11}$. Continued assessment is sometimes necessary, as there is a possibility that late sudden cardiac deaths may be due to heart block. It is clear that the post-procedure course and findings are the critical factors that will determine the need for PPMI for the individual patient.

Other important issues arise regarding PPMI post-TAVR. First, once a PPMI is indicated, what kind of cardiac implanted electronic device (CIED) is appropriate? PPMI implantation is associated with elevated re-hospitalization and mortality risk. ${ }^{12}$ The cause of this observation is unclear, but one could postulate that device related complications including infection could play a role ${ }^{13}$. In general, $2 \%$ of patients implanted with CIED develop a device related infection at 5 years ${ }^{14}$. Intravascular infection associated with a valve prosthesis carries with it a high mortality. Patients with prosthetic valve endocarditis experience a mean hospital stay of 28-33 days and an in-hospital mortality of $22-27 \% .{ }^{15}$ Without pre-existing ventricular dysfunction and sinus node dysfunction, a VDI leadless pacemaker may be sufficient to maintain AV synchrony and reduce risk of CIED related infection. For those with second degree AV block or high degree AV block post TAVR, RV pacing requirements may be low and decrease over time. Thus, a VVI leadless pacemaker may also be sufficient.

Conversely, reduction in infection risk by implanting a leadless pacemaker may be offset by the potentially deleterious effects of right ventricular pacing on ventricular systolic function. ${ }^{16}$ Similarly, left bundle branch 
block may also be associated with development of left ventricular systolic dysfunction. Initially, the development of left bundle branch block was not considered to affect morbidity and mortality post TAVR. However, recent data suggest that mortality is worse with new-onset LBBB post TAVR. ${ }^{17}$ The impacts of cardiac dyssynchrony, via right ventricular pacing or LBBB, on long-term post-TAVR outcomes is unclear. Also unclear, is whether or not early physiologic pacing would benefit this group of patients. In the setting of LBBB and preserved left ventricular ejection fraction, cardiac resynchronization therapy (CRT) has not been shown to have meaningful improvement in outcomes. ${ }^{18}$ The timing of CRT implantation for those with LBBB and systolic dysfunction post-TAVR also requires further investigation, since the effects of cardiac dyssynchrony are likely to be higher in this group.

On the horizon, leadless absorbable temporary pacing is an emerging technology that could change the way we approach risk stratification of post-TAVR patients to further limit morbidity associated with pacing. ${ }^{19}$ Most cardiac conduction abnormalities improve or present within 48 hours, but some patients resolve their cardiac conduction or present with high degree AV block beyond this period ${ }^{20,21}$. For patients requiring percutaneous trans-venous temporary pacing in the perioperative period, observation in the ICU with temporary pacing in a geriatric population comes with its own hazard, namely, hospital-associated functional decline. ${ }^{22}$ Preoperative frailty in this patient populace also influences this outcome. Even a pectoral wound and the shoulder restrictions required to prevent lead dislodgment can conflict with the moderate assistance measures to allow mobility. Leadless absorbable temporary pacing could allow a longer observation period to decide whether PPMI is necessary enabling early hospital discharge and early mobilization.

In conclusion, while pre-procedure risk factors for PPMI following TAVR as outlined in this meta-analysis are important for determining procedural approaches, including choice of valve, and the risk for PPMI, many questions about the rhythm management of these patients remain unanswered and will require continual re-evaluation as TAVR technology continues to evolve.

\section{References}

1. Schwarz F, Baumann P, Manthey J, et al. The effect of aortic valve replacement on survival. Circulation 1982;66:1105-10.

2. Alivizatos PA. Dwight Emary Harken, MD, an all-American surgical giant: Pioneer cardiac surgeon, teacher, mentor. Baylor University Medical Center Proceedings 2018;31:554-7.

3. Eveborn GW, Schirmer H, Heggelund G, Lunde P, Rasmussen K. The evolving epidemiology of valvular aortic stenosis. The Troms $\varnothing$ Study. Heart 2013;99:396-400.

4. Gannon PG, Sellers RD, Kanjuh VI, Edwards JE, Lillehei CW. Complete heart block following replacement of the aortic valve. Circulation 1966;33:I152-I61.

5. Rosato S, Biancari F, D'Errigo P, et al. One-Year Outcomes after Surgical versus Transcatheter Aortic Valve Replacement with Newer Generation Devices. Journal of Clinical Medicine 2021;10:3703.

6. Leon MB, Smith CR, Mack MJ, et al. Transcatheter or Surgical Aortic-Valve Replacement in IntermediateRisk Patients. New England Journal of Medicine 2016;374:1609-20.

7. Mack MJ, Leon MB, Thourani VH, et al. Transcatheter Aortic-Valve Replacement with a BalloonExpandable Valve in Low-Risk Patients. New England Journal of Medicine 2019;380:1695-705.

8. Wang T, Ou A, Xia P, Tian J, Wang H, Cheng Z. Predictors for the risk of permanent pacemaker implantation after transcatheter aortic valve replacement: a systematic review and meta-analysis Journal of Cardiac Surgery 2021.

9. Kawashima T, Sato F. Visualizing anatomical evidences on atrioventricular conduction system for TAVI. International Journal of Cardiology 2014;174:1-6.

10. Rodés-Cabau J, Ellenbogen KA, Krahn AD, et al. Management of Conduction Disturbances Associated With Transcatheter Aortic Valve Replacement: JACC Scientific Expert Panel. J Am Coll Cardiol 
2019;74:1086-106.

11. Ferreira T, Da Costa A, Cerisier A, et al. Predictors of high-degree conduction disturbances and pacemaker implantation after transcatheter aortic valve replacement: Prognostic role of the electrophysiological study. Pacing and Clinical Electrophysiology 2021;44:843-55.

12. Nazif TM, Dizon M, Jose, Hahn RT, et al. Predictors and Clinical Outcomes of Permanent Pacemaker Implantation After Transcatheter Aortic Valve Replacement. JACC: Cardiovascular Interventions 2015;8:609 .

13. Clementy N, Bisson A, Bodin A, et al. Outcomes associated with pacemaker implantation following transcatheter aortic valve replacement: A nationwide cohort study. Heart Rhythm 2021.

14. Cahill TJ, Prendergast BD. Infective endocarditis. The Lancet 2016;387:882-93.

15. Lalani T. In-Hospital and 1-Year Mortality in Patients Undergoing Early Surgery for Prosthetic Valve Endocarditis. JAMA Internal Medicine 2013;173:1495.

16. Sweeney MO, Hellkamp AS, Ellenbogen KA, et al. Adverse Effect of Ventricular Pacing on Heart Failure and Atrial Fibrillation Among Patients With Normal Baseline QRS Duration in a Clinical Trial of Pacemaker Therapy for Sinus Node Dysfunction. Circulation 2003;107:2932-7.

17. Regueiro A, Abdul-Jawad Altisent O, Del Trigo M, et al. Impact of New-Onset Left Bundle Branch Block and Periprocedural Permanent Pacemaker Implantation on Clinical Outcomes in Patients Undergoing Transcatheter Aortic Valve Replacement. Circulation: Cardiovascular Interventions 2016;9:e003635.

18. Curtis AB, Worley SJ, Adamson PB, et al. Biventricular Pacing for Atrioventricular Block and Systolic Dysfunction. New England Journal of Medicine 2013;368:1585-93.

19. Choi YS, Yin RT, Pfenniger A, et al. Fully implantable and bioresorbable cardiac pacemakers without leads or batteries. Nature Biotechnology 2021;39:1228-38.

20. Kaplan RM, Yadlapati A, Cantey EP, et al. Conduction recovery following pacemaker implantation after transcatheter aortic valve replacement. Pacing and Clinical Electrophysiology 2018.

21. Ream K, Sandhu A, Valle J, et al. Ambulatory Rhythm Monitoring to Detect Late High-Grade Atrioventricular Block Following Transcatheter Aortic Valve Replacement. Journal of the American College of Cardiology 2019;73:2538-47.

22. Cohen Y, Zisberg A, Chayat Y, et al. Walking for Better Outcomes and Recovery: The Effect of WALK-FOR in Preventing Hospital-Associated Functional Decline Among Older Adults. The Journals of Gerontology: Series A 2019;74:1664-70.

1. Stewart BF, Siscovick D, Lind BK, et al. Clinical Factors Associated With Calcific Aortic Valve Disease. Journal of the American College of Cardiology 1997;29:630-4.

2. Alivizatos PA. Dwight Emary Harken, MD, an all-American surgical giant: Pioneer cardiac surgeon, teacher, mentor. Baylor University Medical Center Proceedings 2018;31:554-7.

3. Eveborn GW, Schirmer H, Heggelund G, Lunde P, Rasmussen K. The evolving epidemiology of valvular aortic stenosis. The Tromso Study. Heart 2013;99:396-400.

4. Gannon PG, Sellers RD, Kanjuh VI, Edwards JE, Lillehei CW. Complete heart block following replacement of the aortic valve. Circulation 1966;33:I152-I61.

5. Rosato S, Biancari F, D'Errigo P, et al. One-Year Outcomes after Surgical versus Transcatheter Aortic Valve Replacement with Newer Generation Devices. Journal of Clinical Medicine 2021;10:3703.

6. Leon MB, Smith CR, Mack MJ, et al. Transcatheter or Surgical Aortic-Valve Replacement in Intermediate-Risk Patients. New England Journal of Medicine 2016;374:1609-20. 
7. Mack MJ, Leon MB, Thourani VH, et al. Transcatheter Aortic-Valve Replacement with a BalloonExpandable Valve in Low-Risk Patients. New England Journal of Medicine 2019;380:1695-705.

8. Wang T, Ou A, Xia P, Tian J, Wang H, Cheng Z. Predictors for the risk of permanent pacemaker implantation after transcatheter aortic valve replacement: a systematic review and meta-analysis Journal of Cardiac Surgery 2021.

9. Kawashima T, Sato F. Visualizing anatomical evidences on atrioventricular conduction system for TAVI. International Journal of Cardiology 2014;174:1-6.

10. Rodes-Cabau J, Ellenbogen KA, Krahn AD, et al. Management of Conduction Disturbances Associated With Transcatheter Aortic Valve Replacement: JACC Scientific Expert Panel. J Am Coll Cardiol 2019;74:1086-106.

11. Ferreira T, Da Costa A, Cerisier A, et al. Predictors of high-degree conduction disturbances and pacemaker implantation after transcatheter aortic valve replacement: Prognostic role of the electrophysiological study. Pacing and Clinical Electrophysiology 2021;44:843-55.

12. Nazif TM, Dizon M, Jose, Hahn RT, et al. Predictors and Clinical Outcomes of Permanent Pacemaker Implantation After Transcatheter Aortic Valve Replacement. JACC: Cardiovascular Interventions 2015;8:609 .

13. Clementy N, Bisson A, Bodin A, et al. Outcomes associated with pacemaker implantation following transcatheter aortic valve replacement: A nationwide cohort study. Heart Rhythm 2021.

14. Cahill TJ, Prendergast BD. Infective endocarditis. The Lancet 2016;387:882-93.

15. Lalani T. In-Hospital and 1-Year Mortality in Patients Undergoing Early Surgery for Prosthetic Valve Endocarditis. JAMA Internal Medicine 2013;173:1495.

16. Sweeney MO, Hellkamp AS, Ellenbogen KA, et al. Adverse Effect of Ventricular Pacing on Heart Failure and Atrial Fibrillation Among Patients With Normal Baseline QRS Duration in a Clinical Trial of Pacemaker Therapy for Sinus Node Dysfunction. Circulation 2003;107:2932-7.

17. Regueiro A, Abdul-Jawad Altisent O, Del Trigo M, et al. Impact of New-Onset Left Bundle Branch Block and Periprocedural Permanent Pacemaker Implantation on Clinical Outcomes in Patients Undergoing Transcatheter Aortic Valve Replacement. Circulation: Cardiovascular Interventions 2016;9:e003635.

18. Curtis AB, Worley SJ, Adamson PB, et al. Biventricular Pacing for Atrioventricular Block and Systolic Dysfunction. New England Journal of Medicine 2013;368:1585-93.

19. Choi YS, Yin RT, Pfenniger A, et al. Fully implantable and bioresorbable cardiac pacemakers without leads or batteries. Nature Biotechnology 2021;39:1228-38.

20. Kaplan RM, Yadlapati A, Cantey EP, et al. Conduction recovery following pacemaker implantation after transcatheter aortic valve replacement. Pacing and Clinical Electrophysiology 2018.

21. Ream K, Sandhu A, Valle J, et al. Ambulatory Rhythm Monitoring to Detect Late High-Grade Atrioventricular Block Following Transcatheter Aortic Valve Replacement. Journal of the American College of Cardiology 2019;73:2538-47.

22. Cohen Y, Zisberg A, Chayat Y, et al. Walking for Better Outcomes and Recovery: The Effect of WALK-FOR in Preventing Hospital-Associated Functional Decline Among Older Adults. The Journals of Gerontology: Series A 2019;74:1664-70.

Figure. ECG and electrophysiology recordings from a 76 year old woman who underwent TAVR with an Edwards S3 valve. Post-TAVR ECGs showed PR prolongation of $30 \mathrm{~ms}$ and a left bundle branch block (LBBB). At electrophysiology study, Panel A shows the LBBB was intermittent in sinus rhythm. In panel $\mathrm{B}$, there is no difference in $\mathrm{HV}$ interval between the first beat with LBBB and the second beat without LBBB 
(HV was $55 \mathrm{~ms}$ ). Panel $\mathrm{C}$ shows 6 beats of atrial pacing at $100 \mathrm{bpm}$ with normal conduction (no LBBB). When pacing stops, sinus rhythm resumes at a much lower rate and the LBBB resumes. This phenomenon of bradycardia-dependent LBBB is known as phase 4 block. Based on these findings a pacemaker was not recommended.
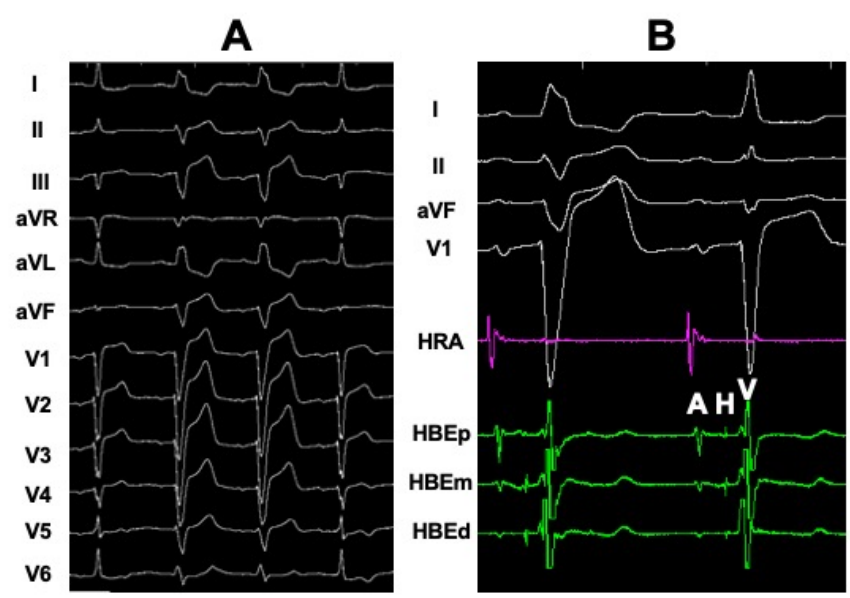